\title{
Structural and molecular basis of cross-seeding barriers in amyloids
}

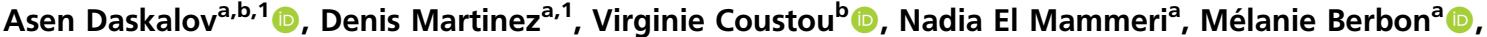

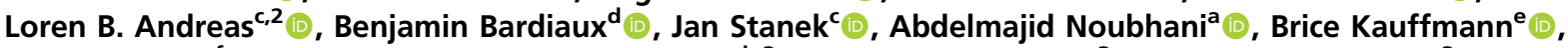

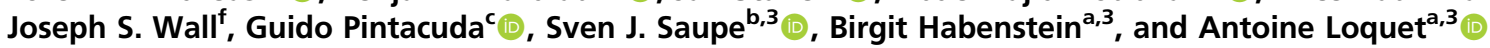

${ }^{a}$ CNRS, Chemistry and Biology of Membranes and Nanoobjects (CBMN) UMR 5348, Institut Europeen de Chimie et Biologie (IECB), University of Bordeaux, F-33600 Pessac, France; ${ }^{b}$ CNRS, Institut de Biochimie et Genetique Cellulaires (IBGC) UMR 5095, University of Bordeaux, F-33077 Bordeaux, France; ${ }^{\circ}$ Centre de Résonance Magnétique Nucléaire à Très Hauts Champs, Université de Lyon (CNRS/Ecole Normale Superieure de Lyon/Université Claude Bernanrd-Lyon 1), F-69100 Villeurbanne, France; dStructural Bioinformatics Unit, CNRS UMR 3528, Institut Pasteur, F-75015 Paris, France; ${ }^{2}$ CNRS, INSERM, IECB, Unité Mixte de Service 3033, University of Bordeaux, F-33600 Pessac, France; and fDepartment of Biology, Brookhaven National Laboratory, Upton, NY $11973-5000$

Edited by David S. Eisenberg, David Geffen School of Medicine at University of California, Los Angeles, CA, and approved November 16, 2020 (received for review July 5,2020$)$

Neurodegenerative disorders are frequently associated with $\beta$-sheet-rich amyloid deposits. Amyloid-forming proteins can aggregate under different structural conformations known as strains, which can exhibit a prion-like behavior and distinct pathophenotypes. Precise molecular determinants defining strain specificity and cross-strain interactions (cross-seeding) are currently unknown. The HET-s prion protein from the fungus Podospora anserina represents a model system to study the fundamental properties of prion amyloids. Here, we report the amyloid prion structure of HELLF, a distant homolog of the model prion HET-s. We find that these two amyloids, sharing only $17 \%$ sequence identity, have nearly identical $\beta$-solenoid folds but lack cross-seeding ability in vivo, indicating that prion specificity can differ in extremely similar amyloid folds. We engineer the HELLF sequence to explore the limits of the sequence-to-fold conservation and to pinpoint determinants of cross-seeding and prion specificity. We find that amyloid fold conservation occurs even at an exceedingly low level of identity to HET-s (5\%). Next, we derive a HELLF-based sequence, termed HEC, able to breach the cross-seeding barrier in vivo between HELLF and HET-s, unveiling determinants controlling cross-seeding at residue level. These findings show that virtually identical amyloid backbone structures might not be sufficient for cross-seeding and that critical side-chain positions could determine the seeding specificity of an amyloid fold. Our work redefines the conceptual boundaries of prion strain and sheds light on key molecular features concerning an important class of pathogenic agents.

amyloid | prion | sequence to fold | cross-seeding

nuclear magnetic resonance

A myloid-forming proteins undergo a phase transition to form insoluble, polymeric assemblies, which can self-propagate in vivo as prions (1-3). Amyloid aggregates associated with neurodegenerative diseases (i.e., Alzheimer's, Parkinson's, etc.) have a prion-like behavior $(4,5)$ and can propagate under different structural conformations also known as prion "strains" (conformational variants of identical protein sequence), which may be associated with distinct phenotypes of the pathology (6-9). The cross-talk between an infectious amyloid conformation (prion strain) and a naive homologous or heterologous amyloidogenic sequence, referred to as "cross-seeding" $(10,11)$, is a critical event in prion biology, representing the key aspect of infectivity. Amyloid cross-seeding could play a role in the etiology (12) and pathogenesis of various proteinopathies $(13,14)$. However, our understanding of the precise molecular and structural determinants allowing or limiting cross-seeding remains poor.

The fungal HET-s protein constitutes a highly favorable system to study the fundamental properties of prion amyloids, as a high-resolution structure of the propagative prion state is available $(15,16)$. The prion-forming domain (PFD) of HET-s contains two 21 amino acid pseudorepeats (R1 and R2), which are alternately stacked, each repeat forming four $\beta$-strands, adopting a left-handed $\beta$-solenoid fold $(15,16)$. Noteworthy, several reports have stressed structural similarities between the HET-s amyloid fold and pathological amyloids formed by the human prion protein PrP (17) and tau (18).

Despite the structural similarities with some pathological amyloids, HET-s represents a functional amyloid, which is integral to an immunity-related signal transduction pathway in fungi (19). The $\beta$-solenoid fold ensures signal transduction from an activated NOD-like receptor (NLR) to a downstream execution protein (HET-S, a pore-forming variant of the HET-s prion protein). The fold represents a cell death trigger and functions on the basis of the prion principle $(19,20)$. The structural templating of the HET-S PFD into the amyloid fold triggers the cytotoxicity of the $\alpha$-helical HeLo domain, which induces cell death targeting the plasma membrane (21-23). Signal-transducing amyloids are widespread in fungi (24) and at least five

\section{Significance}

Amyloid folds, while performing functional roles in most domains of life, remain a key factor in the emergence and development of multiple neurodegenerative disorders in humans. The significance of our study is twofold: first, by structurally characterizing highly divergent natural prion amyloids, we uncovered that functional amyloids can evolve in a regime of fold conservation, withstanding extreme sequence diversification. Second, we found that virtually identical amyloid backbone structures might not be sufficient for cross-seeding and that key side-chain positions could determine the seeding specificity of an amyloid fold. This work thus sheds light on the fundamental properties of a major category of pathogenic agents.

Author contributions: A.D., B.B., G.P., S.J.S., B.H., and A.L. designed research; A.D., D.M., V.C., N.E.M., M.B., L.B.A., B.B., J.S., A.N., B.K., J.S.W., G.P., S.J.S., B.H., and A.L. performed research; A.D., D.M., V.C., N.E.M., B.B., G.P., S.J.S., B.H., and A.L. analyzed data; A.D., D.M., S.J.S., B.H., and A.L. wrote the paper; and B.B. implemented and performed structure calculations.

The authors declare no competing interest

This article is a PNAS Direct Submission.

Published under the PNAS license.

${ }^{1}$ A.D. and D.M. contributed equally to this work.

${ }^{2}$ Present address: Max Planck Institute for Biophysical Chemistry, D-37077 Göttingen, Germany.

${ }^{3}$ To whom correspondence may be addressed. Email: sven.saupe@ibgc.cnrs.fr, b.habenstein@ iecb.u-bordeaux.fr, or a.loquet@iecb.u-bordeaux.fr.

This article contains supporting information online at https://www.pnas.org/lookup/suppl/ doi:10.1073/pnas.2014085118/-/DCSupplemental.

Published December 21, 2020 
subfamilies of HET-s-related amyloid motifs (HRAMs) have been identified (25). The HRAMs exhibit the two pseudorepeats organization of HET-s and a specific pattern of hydrophobic and polar amino acids, while each subfamily is being defined by the conservation of a set of HRAM-specific residues (25). It has been speculated that the HRAMs may share a common $\beta$-solenoid fold and that natural diversification is driven to preserve specificity of the signaling pathways (between NLRs and cognate effectors) by limiting amyloid cross-seeding between distinct HRAMs $(19,25)$. Here, we use the HRAMs as an experimental framework to investigate 1) the sequence-to-fold relation in prion amyloids and 2) the molecular determinants defining prion specificity, which allow or prevent cross-seeding.

\section{Results}

Molecular Characterization of HELLF. We chose to work with a newly identified HELLF protein, encoded in the genome of Podospora anserina (SI Appendix, Fig. S1), the natural host of the [Het-s] prion. HELLF consists of an N-terminal HELL (HeLo-like) domain and a putative C-terminal PFD. The HELLF PFD carries strongly divergent HRAM pseudorepeats (R1 and R2) showing only $17 \%$ of sequence identity with the PFD repeats of HET-S/s (Fig. $1 A$ and SI Appendix, Fig. S1). HELLF pseudorepeats belong to the HRAM5 family (Fig. $1 B$ ). Strains expressing HELLF(209-277) showed phenotypic bistability exhibiting either a $\left[\Phi^{*}\right]$ phenotype, where the protein remains soluble or a $[\Phi]$ phenotype with formation of dot-like aggregates (Fig. $1 C$ and SI Appendix, Fig. S1). The $[\Phi]$ strains triggered cell death upon anastomosis (cellular fusion) with strains expressing full-length HELLF, while $\left[\Phi^{*}\right]$ strains formed viable heterokaryons (Fig. $1 D$ ). During the cell death reaction, as described for HET-S (22), HELLF relocates to the cell membrane (SI Appendix, Fig. S1). The $\left[\Phi^{*}\right]$ strains (aggregate free) switched spontaneously ( $\sim 24 \mathrm{~h})$ to $[\Phi]$ strains (with aggregates), which in turn could be cured (or reversed) back to the $\left[\Phi^{*}\right]$ state through a sexual cross (SI Appendix, Tables S1 and S2). These results establish HELLF as a distant HET-S homolog and demonstrate that the HRAM5 pseudorepeats bearing PFD of HELLF behave as a prion in $P$. anserina.
Solid-State NMR (ssNMR) Structure of HELLF Prion Domain. We engaged in the structural characterization of HELLF(209-277). The protein self-assembled into unbranched fibrils in vitro (Fig. $1 E$ ), exhibiting a typical cross- $\beta$ signature by $\mathrm{X}$-ray diffraction (SI Appendix, Fig. S2). We took advantage of recent developments for fast magic-angle spinning (MAS) NMR probes (26-28) to establish, and implement for the first time on a fibril sample, a three-dimensional (3D) structure determination approach, based entirely on ${ }^{1} \mathrm{H}-{ }^{1} \mathrm{H}$ proximities. The approach, reminiscent of nuclear Overhauser effect (nOe)-based solution NMR methods to solve soluble globular protein structures, allows a tremendous gain in sensitivity and enables the use of simplified labeling schemes and minimal sample quantities.

Approximately $300 \mu \mathrm{g}$ of fully protonated HELLF(209-277) was packed into a $0.7-\mathrm{mm}$ ssNMR rotor (Fig. $2 A$ ). Ultra-fast MAS performed at rates of $\sim 110 \mathrm{kHz}$ allowed the acquisition of high-resolution ${ }^{1} \mathrm{H}$-detected multidimensional spectra even in fully protonated samples (Fig. $2 B$ and SI Appendix, Fig. S3) with ${ }^{1} \mathrm{H}$ line widths of $\sim 150$ to $200 \mathrm{~Hz}$ enabling assignment of backbone and side-chain protons (29). A unique set of resonances revealed the presence of a single conformational polymorph in the fibrillar assembly, and conformation-dependent chemical shifts (30) revealed a $\beta$-rich rigid core extending from residue Q221 to S272, including eight $\beta$-strands (SI Appendix, Fig. S4). A flexible linker segment (G240-D247), comprising a $\beta$-breaker GxxxPG motif, subdivides the rigid core in two regions with four $\beta$-strands each (SI Appendix, Fig. S4). We employed a combination of $3 \mathrm{D} \mathrm{H}(\mathrm{H}) \mathrm{CH}$ and $\mathrm{H}(\mathrm{H}) \mathrm{NH}$ experiments (27) on a fully protonated, uniformly ${ }^{13} \mathrm{C},{ }^{15} \mathrm{~N}$-labeled sample to derive 178 internuclear ${ }^{1} \mathrm{H}-{ }^{1} \mathrm{H}$ distances (Fig. 2C). The 3D amyloid architectures render the distinction between intra- and intermolecular contacts in ssNMR experiments difficult and usually require complex labeling schemes $(16,31)$. We designed an asymmetric labeling strategy based on an equimolar mixture of fully protonated proteins at natural abundance, randomly coaggregated with deuterated, extensively amide-reprotonated, and uniformly ${ }^{15} \mathrm{~N}$-labeled proteins $\left\{\right.$ scheme denoted as $(1 / 1)\left[\left(\mathrm{U}_{-}{ }^{1} \mathrm{H},{ }^{14} \mathrm{~N}\right) /(\mathrm{U}\right.$ $\left.\left.\left.{ }^{1} \mathrm{H}^{\mathrm{N}},{ }^{2} \mathrm{H},{ }^{15} \mathrm{~N}\right)\right]\right\}$ (Fig. 2E). Using this scheme, we observed 33 intermolecular ${ }^{1} \mathrm{H}-{ }^{1} \mathrm{H}$ interstrand aliphatic-to-amide distances

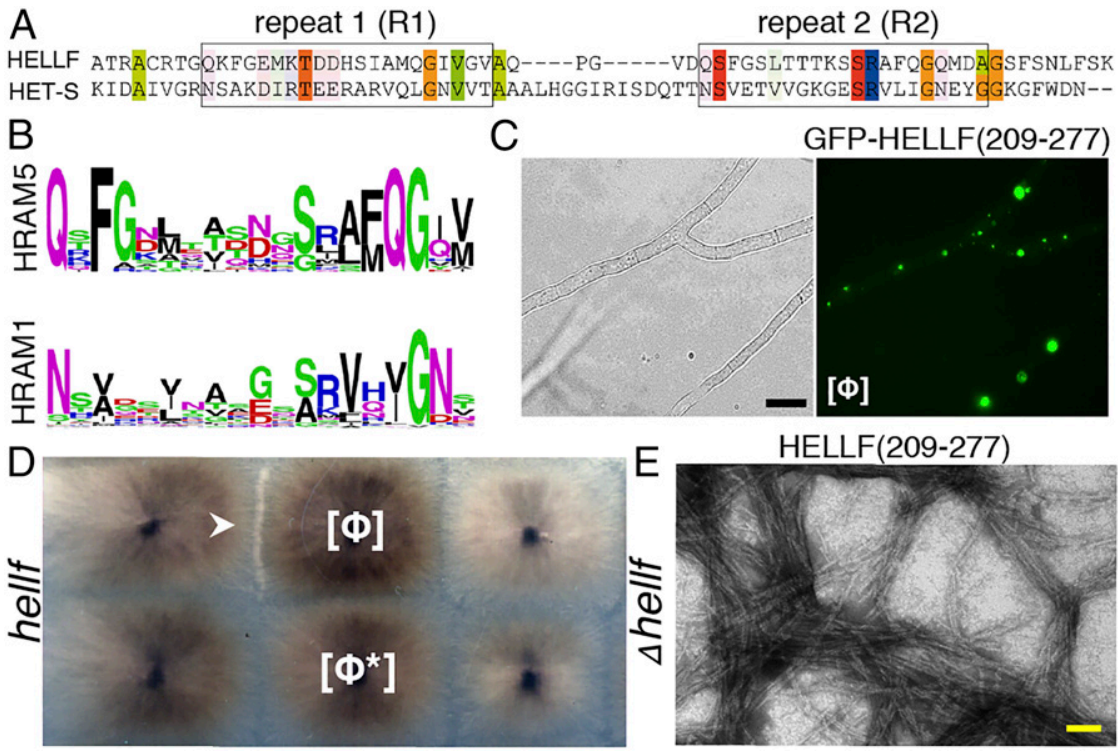

Fig. 1. HELLF is a distant HET-s homolog encoded in the genome of $P$. anserina. (A) Sequence similarity between HELLF and HET-s PFDs. The two pseudorepeats are boxed. Conserved residues are shown in Taylor color scheme and intensity of color reflects degree of conservation. ( $B$ ) MEME cartoons showing the conservation of HRAM-specific residues. $(C)$ Dot-like aggregates of the HELLF PFD [GFP-HELLF(209-277)] formed in $P$. anserina. (Scale bar: $5 \mu$ m.) $(D)$ HELLF controls a programmed cell death reaction as determined by the barrage phenotype (white arrowhead) between strains expressing full-length HELLF and strains expressing the HELLF PFD in the $[\varphi]$ prion state. $(E)$ Electron micrograph of fibrillar assemblies of the HELLF PFD. (Scale bar: 100 nm.) 

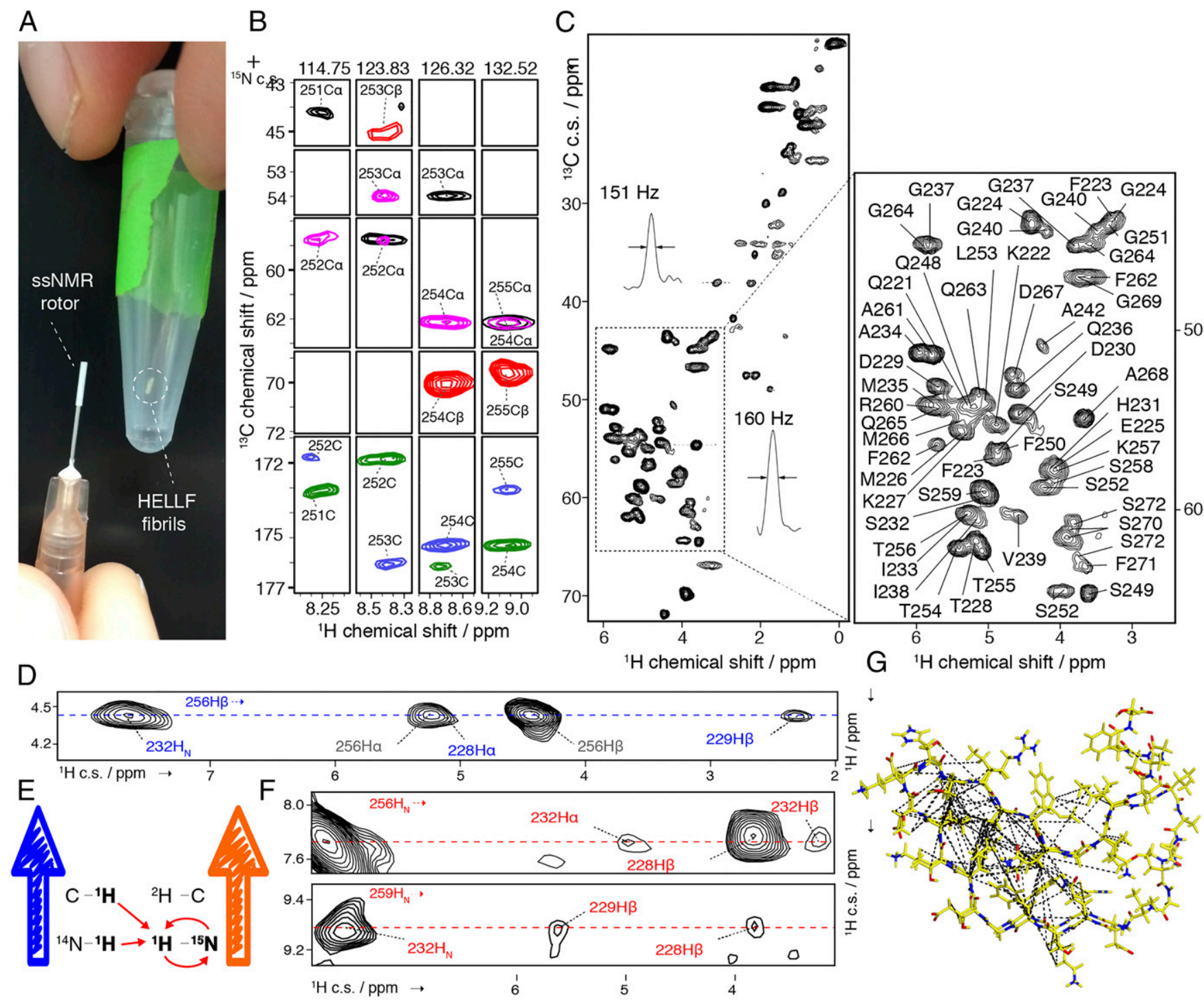

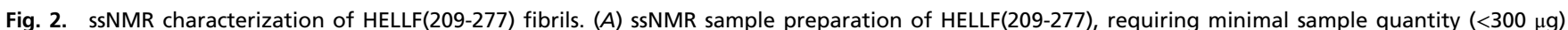
using a 0.7 -mm ssNMR rotor. (B) Extracts of ssNMR spectra for ${ }^{1} \mathrm{H},{ }^{13} \mathrm{C}$, and ${ }^{15} \mathrm{~N}$ sequential assignments. A combination of (HCA) $\mathrm{CB}(\mathrm{CA}) \mathrm{NH}(\mathrm{red})$, (HCO)CA(CO) $\mathrm{NH}$ (black), (H)CANH (purple), (H)CONH (green), and (H)CO(CA)NH (blue) was used to assign HELLF(209-277) fibrils. $(C){ }^{1} \mathrm{H}-{ }^{13} \mathrm{C} s \mathrm{NMMR}$ spectra of fully protonated HELLF(209-277) amyloid fibrils. (D-F) Collection of ssNMR distance restraints. (D) Intramolecular distances based on a $\mathrm{H} \ldots(\mathrm{H}) \mathrm{CH}$ experiment on a fully protonated HELLF sample. (E) ssNMR approach to detect intermolecular interactions, based on a $(1 / 1)\left[\left(\mathrm{U}-{ }^{1} \mathrm{H} /{ }^{14} \mathrm{~N}\right) /\left(\mathrm{U}-{ }^{1} \mathrm{H}_{\mathrm{N}} /{ }^{2} \mathrm{H} /{ }^{15} \mathrm{~N}\right)\right]-$ labeled $\mathrm{HELLF}$ sample. $(F)$ Intermolecular distances based on a $\mathrm{H}$...(H)NH experiment on $(1 / 1)\left[\left(\mathrm{U}-{ }^{1} \mathrm{H} /{ }^{14} \mathrm{~N}\right) /\left(\mathrm{U}-{ }^{1} \mathrm{H}_{\mathrm{N}} /{ }^{2} \mathrm{H} /{ }^{15} \mathrm{~N}\right)\right]$-labeled $\mathrm{HELLF}(209-277)$. (G) ssNMR restraints shown on the HELLF(209-277) rigid core.

relying on a single $3 \mathrm{D} \mathrm{H}(\mathrm{H}) \mathrm{NH}$ spectrum (Fig. $2 D$ ) with an asymmetric polarization transfer (Fig. $2 F$ ). We identified 211 distance restraints in total, of which 176 are long-range restraints $(|i-j|>4)($ Fig. $2 G)$ to derive a 3D structure of the HELLF fibrillar assembly at atomic resolution with 20-conformer bundle r.m.s.d. of $0.73 \AA$ for backbone atoms and $1.18 \AA$ for heavy atoms (Fig. 3 and SI Appendix, Table S3). Our 3D structure determination approach based on ${ }^{1} \mathrm{H}-{ }^{1} \mathrm{H}$ proximities compares favorably to benchmark studies of the HET-s prion domain amyloid structure by ssNMR (16); we detected $\sim 4.5$ structurally meaningful restraints per residue in the rigid amyloid core, a number approaching those used in high-resolution structure determination protocols in solution NMR.

HELLF and HET-s Prions Share an Identical Backbone $\beta$-Solenoid Core. The HELLF fibrillar architecture shows a $\beta$-solenoid fold, made by the intermolecular packing of HELLF monomers along the fibril axis (Fig. 3). The intramolecular HELLF fold is composed of eight $\beta$-strand structural elements separated by a short unstructured region (Gly240-Asp247) (Fig. 3). Intramolecular ssNMR restraints reveal a regular and rigid core, constituted by alternate stacking of R1 (R1 $\beta_{1}$ to $\left.\mathrm{R} 1 \beta_{4}\right)$ and R2 (R2 $\beta_{1}$ to $\left.22 \beta_{4}\right)$ pseudorepeat regions (Figs. $4 B$ and 3). Each pseudorepeat adopts a triangular shape, stabilized by hydrophobic side chains interlaced (SI Appendix, Fig. S5A) inside the amyloid core and protected from the solvent. Water molecules are excluded from the amyloid core (SI Appendix, Fig. S5 $B$ and C). Several $\beta$-breaker glycine residues allow for bending two consecutive $\beta$-arcs into the triangular arrangement of the amyloid core (Fig. $4 B$ ). R1 and R2 repeats are stacked through a hydrogen bond-rich pairing between $\beta$-sheets (R1 $\beta_{\mathrm{i}}$ with $\mathrm{R} 2 \beta_{\mathrm{i}}, \mathrm{T} 255 / \mathrm{S} 232$ and T228/S259). To corroborate the HELLF intramolecular R1/R2 stacking, we 

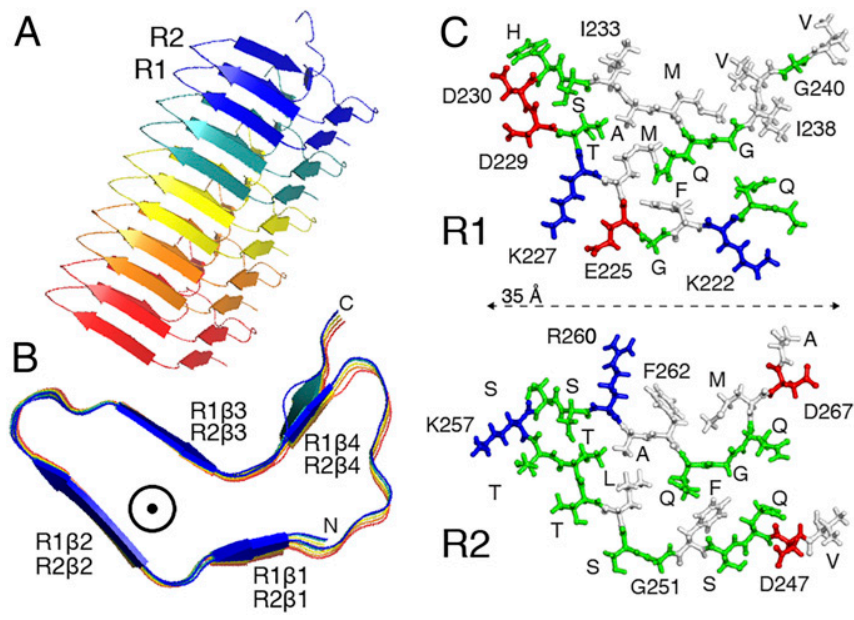

Fig. 3. Solid-state NMR structure of $\operatorname{HELLF}(209-277)$ amyloid assembly. (A) Side view of a ribbon representation of HELLF(209-277) structure, representing five monomers stacked in the fibrillar arrangement. Individual molecules are represented in different colors. $(B, T o p)$ View of the ssNMR $\operatorname{HELLF}(209-277)$ amyloid structure with main fibril axis and labeled $\beta$-strands. (C, Top) View of the solenoid-forming pseudorepeats, R1 (Top) and R2 (Bottom), of HELLF(209-277). Hydrophobic residues are shown in white, acidic residues in red, basic residues in blue, and others in green.

performed scanning transmission electron microscopy (STEM) mass-per-length (MPL) measurements and determined a MPL of $0.99 \pm 0.10 \mathrm{kDa} / \AA ̊$ corresponding to $1.1 \pm 0.1$ molecules per $0.94 \mathrm{~nm}$ (SI Appendix, Fig. S6). Considering a $\beta$-strand repetition of $0.47 \mathrm{~nm}$ in the cross- $\beta$-architecture as measured by X-ray diffraction (SI Appendix, Fig. S2), it leads to a fibril layer (i.e., per $0.47 \mathrm{~nm}$ ) composed of half of a HELLF molecule, in agreement with the fold determined by ssNMR (Figs. $2 G$ and 3 ). The intersubunit packing consists of parallel, pseudo in-register stacking and the overall intermolecular arrangement is consistent with cross- $\beta$-stacked solenoid architecture. Despite the low sequence identity between HET-s and HELLF PFDs, both prion domains adopt virtually identical backbone conformations (Fig. $4 A$ and $C$ ).

We took advantage of the high-resolution structures of HELLF and HET-s to perform molecular modeling on the remaining HRAM families identified in fungal genomes (Fig. $4 E$ ). We found that the observed sequence diversity in the HRAM superfamily is indeed compatible with a unique structural solution underlying this amyloid fold (Fig. $4 E$ ). The findings underscore the importance of the previously identified pattern of hydrophobic and polar amino acids as a minimal requirement to adopt the HET-s-like $\beta$-solenoid fold (25).

In addition, we designed a protein sequence termed HED (HET-s distant) carrying two identical repeats that share less than 5\% identity with HET-s (one residue out of 21) (SI Appendix, Fig. S7A and Table S5). HED was able to form a prion in vivo (SI Appendix, Fig. S7 $B-E$ ). In vitro, HED adopts a HET-s-like $\beta$-solenoid structure as seen by ssNMR (SI Appendix, Fig. S7 $F$ and $G$ ), implying a $\beta$-solenoid fold conservation despite the extremely low sequence identity $(5 \%)$ and a very low sequence similarity $(19 \%)$ to HET-s.

HELLF and HET-s Carry Distinct Prion Specificities In Vivo. Considering the structural similarity between HELLF and HET-s prion folds (Fig. $4 A$ ) and that these proteins occur in the same species, we analyzed the cross-seeding of $[\Phi]$ and [Het-s] prion states in vivo (SI Appendix, Table S2). We found that [Ф] strains do not
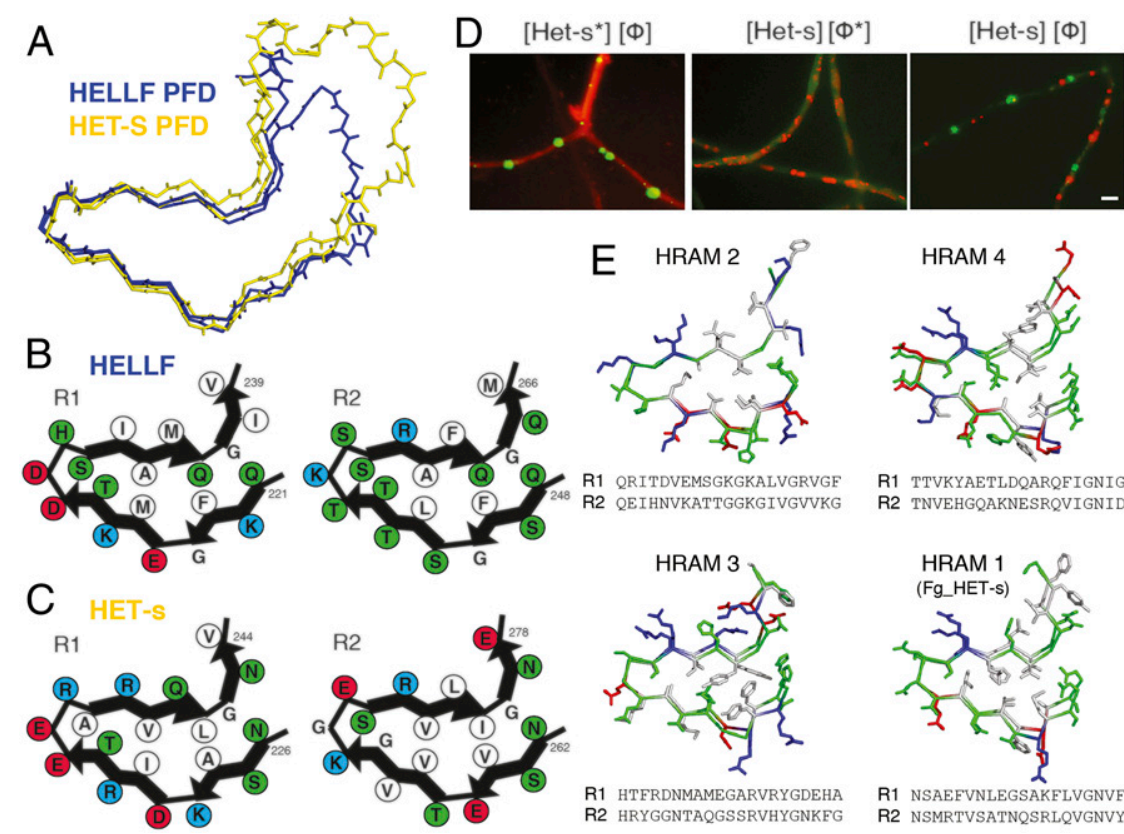
2 QEIHNVKATTGGKGIVGVVKG

R1 TTVKYAETLDQARQEIGNIGL R2 TNVEHGQAKNESRQVIGNIDP
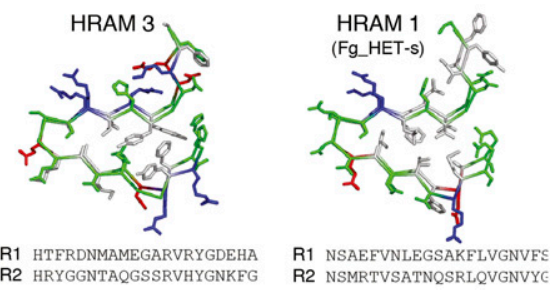

Fig. 4. HELLF(209-277) amyloid fold presents strong similarity with HET-s(218-289), while the two prion domains lack in vivo cross-seeding. ( $A$ ) Backbone structural alignment of HELLF PFD (in blue) and HET-s PFD (in yellow) ssNMR structures. ( $B$ and $C$ ) Four cartoons representing the hydrophobic triangular core of amyloid fibrils formed by the successively stacked pseudorepeats-R1 (Left) and R2 (Right) - of HELLF (B) and of HET-s (C). Amino acid residues decorating the amyloid backbones are drawn as beads of different colors. Hydrophobic residues are shown in white, acidic residues in red, basic residues in blue, and others in green. (D) Fluorescent microscopy images of strains coexpressing HET-s-RFP ([Het-s*] or [Het-s] state) and the cytotoxic-dead HELLF(L52K)-GFP mutant $\left(\left[\Phi^{*}\right]\right.$ or $[\Phi]$ state) exhibiting distinct epigenetic combinations. Full panels are given in SI Appendix, Fig. S8. (Scale bar: $2 \mu \mathrm{m}$.) (E) The 3D models for the two pseudorepeats of different HRAM families. Below the molecular models are shown the protein sequences used to generate the models for each HRAM family. The sequence GenBank/National Center for Biotechnology Information (NCBI) reference IDs are as follows: XP_009252118.1 (HRAM1), CDM29511.1 (HRAM2), XP_007744431.1 (HRAM3), and XP_007838484.1 (HRAM4). 
convert nonprion [Het-s*] strains to the [Het-s] prion state nor do [Het-s] strains induce $[\Phi]$ prion formation (SI Appendix, Table S2). HELLF PFD fibrils show no [Het-s] infectivity and HET-s PFD fibrils show no [ $\Phi]$ infectivity in transfection assays (SI Appendix, Table S4). HELLF and HET-s PFDs form independent aggregates in vivo, indicating that the two prions do not coaggregate (Fig. 4D and SI Appendix, Fig. S8). Strains coexpressing HELLF and HET-s can display four alternate epigenetic states $\left(\left[\right.\right.$ Het-s*] $\left[\Phi^{*}\right]$, [Het-s] [ $\left.\Phi\right]$ but also [Het-s*] $[\Phi]$ and [Het-s] $\left.\left[\Phi^{*}\right]\right)$ confirming that [Het-s] and $[\Phi]$ are independent prions (SI Appendix, Fig. S8 and Table S2). Since the backbone structures in the amyloid fold of HET-s and HELLF PFDs are nearly identical (Fig. 4A), it appears that strong structural similarity and interactions of the cross- $\beta$-backbones are insufficient for amyloid templating and/or coaggregation.

At the level of side-chain packing, HELLF has more hydrophilic $\left(\mathrm{Q}^{221}, \mathrm{Q}^{236}, \mathrm{Q}^{248}\right.$, and $\left.\mathrm{Q}^{263}\right)$ side chains pointing inside the amyloid core compared to HET-s $\left(\mathrm{N}^{226}\right.$ and $\left.\mathrm{N}^{262}\right)$. A striking difference is observed at the end of the third $\beta$-strand, composed of $Q^{240} \mathrm{~L}(\mathrm{R} 1)$ and $\mathrm{L}^{276} \mathrm{I}(\mathrm{R} 2)$ in HET-s and $\mathrm{M}^{235} \mathrm{Q}(\mathrm{R} 1)$ and $\mathrm{F}^{262} \mathrm{Q}(\mathrm{R} 2)$ in HELLF. Therefore, the lack of cross-seeding between HET-s and HELLF could result from unfavorable hydrophobic-hydrophilic packing between solvent exposed HET-s $Q^{240}$ and HELLF $F^{262}$ as well as HET-s $\mathrm{L}^{241}$ and HELLF $\mathrm{Q}^{263}$.

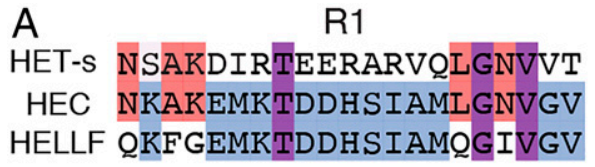

A
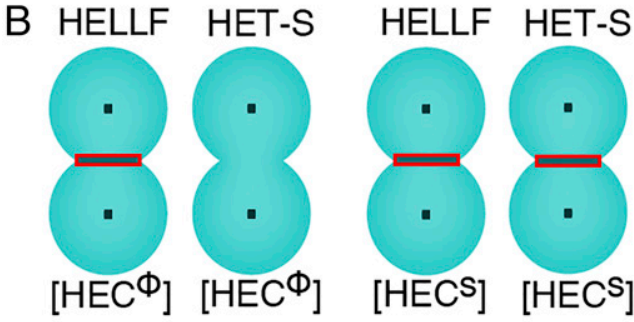

$\mathrm{D}$

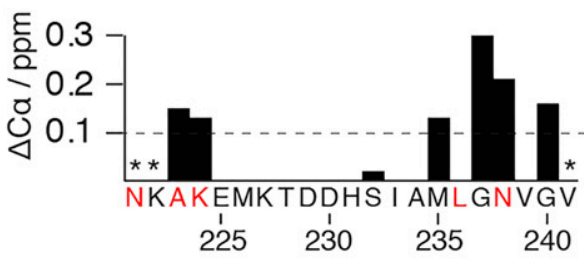

E
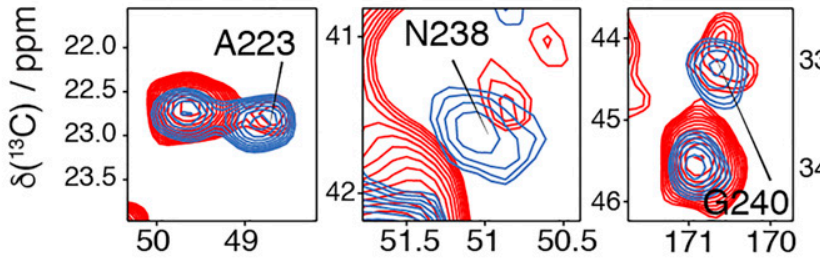

$\mathrm{R} 2$

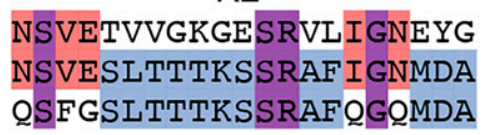

\section{C}
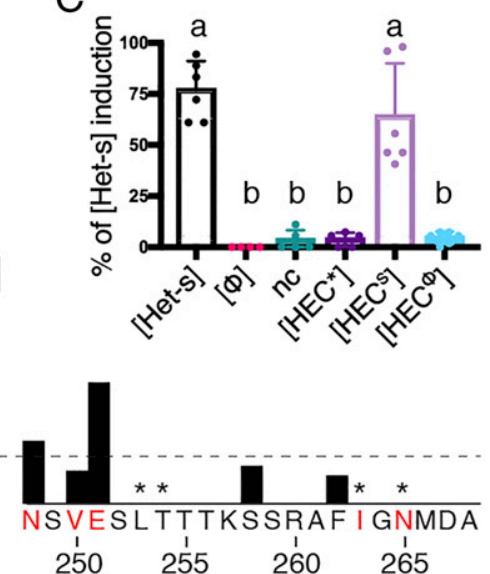

F

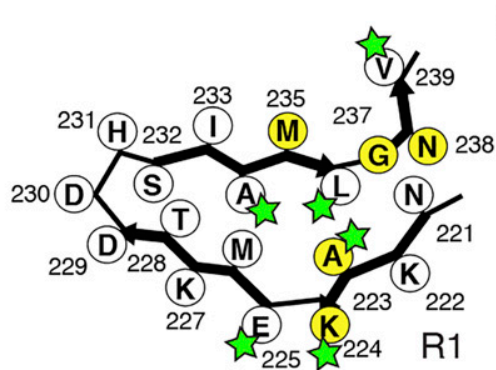

$\delta\left({ }^{13} \mathrm{C}\right) / \mathrm{ppm}$

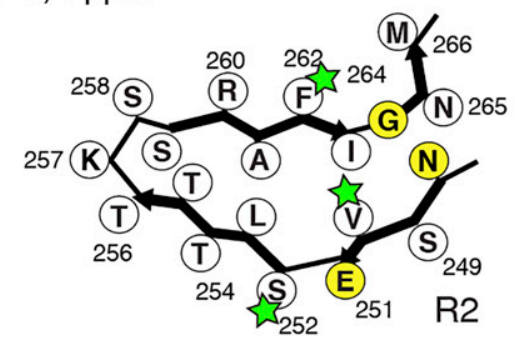

Fig. 5. HELLF-derived chimeric protein HEC breaches the prion cross-seeding barrier between HELLF and HET-s. ( $A$ ) Sequence alignments of the two pseudorepeats (R1 and R2), constituting the PFDs of HELLF, HET-s, and the engineered protein HEC. HELLF-specific residues are shown in blue. HET-s-specific HRAM1-defining residues are shown in red. Residues shared between all three proteins are shown in purple. ( $B$ ) Representation of barrage phenotypes between strains expressing full-length HET-S or HELLF in confrontations with HEC-expressing strains of [HEC ${ }^{\mathrm{S}}$ (carrying $\mathrm{HEC}$ prion strain induced by contact with [Het-s] prion) or $\left[\mathrm{HEC}^{\phi}\right]$ (HEC prion strain induced by [ $\left.\Phi\right]$ ) phenotypes. The barrage reaction is shown as a line separating two incompatible strains (green circles). (C) Induction of the [Het-s] prion by HEC-expressing strains in vivo. [Het-s] induction is measured in percentage of prion-free [Het-s*] strains converted (by the cited strains) to prion-infected [Het-s] strains. Negative control is indicated with a minus sign. Experiments were performed at least in triplicate. $P$ value $(a \neq b)<0.0001$, one-way ANOVA with Tukey's multiple comparisons test. $(D)$ C $\alpha$ chemical shift differences between HEC seeded with [Het-s] and $[\Phi]$. Nonidentical residues between HEC and HELLF are colored in red. Asterisks indicate unassigned residues because of spectral ambiguities. $(E)$ Extracts of ${ }^{13} \mathrm{C}-{ }^{13} \mathrm{C}$ ssNMR spectra of HEC fibrils seeded with [Het-s] (red) or [ $\Phi$ ] (blue). ( $F$ ) Cartoon representation of the amyloid backbone of HEC pseudorepeats. Residues with highest conformational changes between HEC seed by HET-s or HELLF, as measured by difference in chemical shift on C $\alpha$, are shown in yellow. Green stars highlight residues in two conformations. 
HRAM-Specific Residues Control Cross-Seeding between HELLF and HET-s. The results suggested that side-chain residues, decorating the amyloid backbone, could play a key role in defining prion specificity and cross-seeding. Thus, we decided to test whether by varying HRAM-specific residues between HET-s and HELLF PFDs, the cross-seeding barrier between the two prions could be breached. We replaced five residues on each of the two 21 amino acid HRAM5 pseudorepeats of HELLF with residues from the corresponding positions found in the HRAM1 pseudorepeats of HET-s. The engineered sequence was termed HEC (HET-s closer). We targeted for replacement residues that are highly conserved inside each HRAM (Fig. $1 B$ ) and essential for distinguishing HRAM5 (HELLF) from HRAM1 (HET-s) (25) (Fig. 5A). The five amino acid substitutions for each pseudorepeat of HELLF were introduced in two strongly conserved HRAM5-specific submotifs of the protein-the QxFG (position 1 to 4 , where $x$ is any possible amino acid residue) and QG(Q/I) (position 16 to 18 ) submotifs (Figs. $5 A$ and $1 B$ ). The substitution of $\mathrm{Q}$ (glutamine) in the $\mathrm{QG}(\mathrm{Q} / \mathrm{I})$ submotif would also remove one of the unfavorable hydrophobic-hydrophilic interactions in the potential cross-seeded interface.

We expressed HEC in a $\Delta$ het-s $\Delta$ hellf strain and tested the ability of the protein to propagate as a prion and to carry [Het-s] and/or $[\Phi]$ prion specificity in vivo. Strains expressing GFP-HEC showed two distinct phenotypes; [HEC*] strains presented diffuse GFP-HEC fluorescence, did not induce [Het-s] or $[\Phi]$ prions, and were unable to trigger cell death by incompatibility with HET-S- or HELLF-expressing strains (SI Appendix, Fig. S9 and Tables S6 and S7). We observed that some [HEC*] strains did, spontaneously and at low rate $(\sim 10 \%)$, transition into a [HEC] state characterized by the appearance of fluorescent dotlike aggregates (SI Appendix, Fig. S9 and Table S6). None of the spontaneous [HEC] strains were able to induce [Het-s] or produce a barrage with a HET-S strain. Yet, [HEC] strains induced cell death with HELLF-expressing strains, indicating that the engineered HEC sequence shows $[\Phi]$ prion specificity and we termed the phenotype $\left[\mathrm{HEC}^{\Phi}\right]$ (Fig. $4 B$ and SI Appendix, Fig. S9). We were equally able to induce the $\left[\mathrm{HEC}^{\Phi}\right]$ phenotype by exposing [HEC*] strains to $[\Phi]$ strains (SI Appendix, Table S7). Spontaneously formed (or $[\Phi]$ induced) $\left[\mathrm{HEC}^{\Phi}\right]$ strains converted $\left[\mathrm{HEC}^{*}\right]$ strains into a $\left[\mathrm{HEC}^{\Phi}\right]$ prion state after contact, demonstrating the ability of aggregated HEC to self-propagate (behave as prion) (SI Appendix, Fig. S9).

Next, we found that after a contact with a [Het-s] strain, the prion-free [HEC* ${ }^{*}$ strains could equally be converted to a [HEC] state, characterized by the appearance of fluorescent GFP-HEC dot-like aggregates and the ability to induce cell death with HELLF, indicating that the prion state of HEC can be induced both by HELLF and HET-s (SI Appendix, Table S7). Importantly, we found that [Het-s]-induced [HEC] strains, unlike the $\left[\mathrm{HEC}^{\Phi}\right]$ strains, were capable of converting [Het-s*] strains into [Het-s] strains and to induce cell death with HET-S (Fig. $5 B$ and $C$ and SI Appendix, Fig. S9). Hence, we termed this phenotype $\left[\mathrm{HEC}^{\mathrm{S}}\right]$. Importantly, $\left[\mathrm{HEC}^{\Phi}\right]$ and $\left[\mathrm{HEC}^{\mathrm{S}}\right]$ phenotypes remained stable in time and faithfully self-propagated over several passages (SI Appendix, Tables S7 and S8). In addition, we observed that GFP-HEC aggregates from $\left[\mathrm{HEC}^{\mathrm{S}}\right]$ strains partially colocalized with [Het-s] and not with $[\Phi]$, while fluorescent HEC aggregates from the $\left[\mathrm{HEC}^{\Phi}\right]$ strain produced the opposite results and colocalized predominantly with $[\Phi]$ (SI Appendix, Fig. S9). We concluded that the engineered HEC sequence could propagate as two distinct prion strains $\left[\mathrm{HEC}^{\Phi}\right]$ and $\left[\mathrm{HEC}^{\mathrm{S}}\right]$ and was capable of breaching the cross-seeding barrier between [Het-s] and $[\Phi]$ prions. A likely explanation could be found in the reduced hydrophilic-hydrophobic clashes between HEC and the sequences of the two fungal prions (HET-s and HELLF), following the replacement of two strongly conserved hydrophilic HRAM5-specific residues (Q236 and Q263 in HELLF) with
HRAM1-specific hydrophobic residues (L241 and I277 in HETs). Because HEC was designed by the targeted replacement of HRAM-specific residues, we concluded that such residues play a key role in the control of prion propagation, allowing or preventing cross-seeding between HRAM families.

Cross-Seeding Induced Structural Plasticity of HEC Near HRAM-Specific Residues. To pinpoint determinants of the cross-seeding at a structural level, we investigated the engineered protein sequence in vitro using ssNMR. Based on the resonance assignment of recombinant ${ }^{13} \mathrm{C},{ }^{15} \mathrm{~N}$-labeled sample, HEC adopts a cross- $\beta$-fold, highly similar to HET-s and HELLF (SI Appendix, Figs. S10 and $\mathrm{S} 11 B$ ). We then assembled HEC fibrils in vitro in the presence of $5 \%$ unlabeled HET-s or HELLF, respectively termed $\mathrm{HEC}^{\mathrm{S}}$ and $\mathrm{HEC}^{\Phi}$. The resulting fibrillar states were analyzed using solidstate NMR and the detected spectral fingerprints were similar between the three HEC preparations (HEC, HEC ${ }^{\mathrm{S}}$, and $\mathrm{HEC}^{\Phi}$ ), while exhibiting slight structural differences at some amino acid positions (SI Appendix, Fig. S11). Both coaggregation experiments (HEC $+5 \%$ HET-s and HEC $+5 \%$ HELLF) produced relatively similar chemical shift perturbations in HEC, suggesting similar interaction surfaces between the peptides (SI Appendix, Fig. S11) To gain further insight into the structural differences between HEC coaggregated with HET-s or HELLF, we compared both chemical shift sets and plotted the differences as a function of the primary sequence (Fig. 5D). We detected small CA chemical shift variations on residues constituting the amyloid core of $\mathrm{HEC}^{\mathrm{S}}$ and $\mathrm{HEC}^{\Phi}$, suggesting slight conformational changes within the assemblies (Fig. $5 D$ and $E$ ). Remarkably, most of the residues showing the highest backbone chemical shift difference between $\mathrm{HEC}^{\mathrm{S}}$ and $\mathrm{HEC}^{\Phi}$ were part of or adjacent to the HRAM-defining positions that were modified in HELLF to engineer HEC (highlighted in red in Fig. $5 D$ ). Chemical shift differences are not only observed at the level of backbone conformation but also on sidechain NMR signals (e.g., M235 and F262). Additional NMR signals were observed (peak doubling, i.e., A223) between $\mathrm{HEC}^{\mathrm{S}}$ / $\mathrm{HEC}^{\Phi}$ and HEC (Fig. $5 E$ ), indicating a second local structural conformation for some residues (Fig. $5 F$ ). Considering chemical shift differences and presence of peak doubling, we observed that the $\mathrm{QxFG}$ and $\mathrm{QG}(\mathrm{Q} / \mathrm{I})$ submotifs, which distinguish HELLF (HARM5) from HET-s (HRAM1) and have been partially replaced in HELLF to engineer HEC, are the most impacted regions during the cross-seeding experiments of HEC (Figs. 5 and $1 B)$. Thus, the results indicate that the engineered HEC sequence adopts a similar amyloid fold to HET-s and HELLF, while exhibiting limited structural plasticity near several different HRAM-specific residues during cross-seeding. Although these conformational changes are subtle, they are in agreement with the observed HEC prion strains in vivo and highlight the role of HRAM-specific residues as potential hotspots defining prion infectivity and controlling cross-seeding.

\section{Discussion}

Our study exploits the natural diversity occurring in a superfamily of fungal prion domains to document the limits of sequence-to-fold conservation in amyloids and the molecular and structural determinants of cross-seeding. First, by structurally characterizing highly divergent natural prion amyloids of the HRAM family, we uncovered that functional amyloids can evolve in a regime of fold conservation, withstanding extreme sequence diversification. Our work thus indicates that the sequence-to-fold evolutionary interplay for functional amyloids is similar to what has been described for globular (32) and membrane proteins (33) with fold conservation occurring even at very low levels of sequence identity and similarity. These findings are in agreement with previous studies documenting the high resistance of the $\beta$-solenoid fold to an extensive array of amino acids substitutions $(34,35)$. The apparent rigidity of $\beta$-solenoids has been 
proposed as a key feature for this class of proteins to perform as scaffolding devices (36). Here, the robustness of the $\beta$-solenoid fold would equally allow for the diversification of the HRAM signaling specificities (25).

The experimental HELLF ssNMR structure as well as the study of synthetic HRAM-derived proteins (HEC and HED) confirms structural and functional importance of the pattern of hydrophobic/polar side-chain arrangement in HRAM families. This combination of bioinformatics and experimental approaches now opens the possibility to predictably engineer the generic HRAM $\beta$-solenoid fold.

Second, this study sheds light in an unprecedented way on the determinants of amyloid cross-seeding. We found that virtually identical amyloid backbone structures might not be sufficient for cross-seeding and that critical side-chain positions could determine the seeding specificity of an amyloid fold. Extrapolating our conclusions to amyloids causing neurodegeneration, especially considering the structural similarities between the HRAM $\beta$-solenoid fold and $\beta$-solenoid folds of pathological amyloids in humans $(17,18,37,38)$, provides a conceptual update on key features of the molecular behavior for this category of pathogenic agents.

\section{Materials and Methods}

Prion Propagation and Incompatibility Assays. Incompatibility phenotypes were determined by confronting strains of solid corn meal agar medium and a "barrage" reaction was assessed 2 to $3 \mathrm{~d}$ postcontact. Prion propagation was assayed as the ability to transmit the $[\varphi]$ prion phenotype from a $[\varphi]$-donor strain to a $\left[\varphi^{\star}\right]$ prion-free tester strain after confrontation on solid medium. Transformants were confronted to wild-type strains either directly or after contact with a $[\varphi]$-donor strain 6,11 , and $17 \mathrm{~d}$ after transfection to evaluate $\left[\varphi^{\star}\right]$ and $[\varphi]$ phenotype frequencies and spontaneous $[\varphi]$ prion propagation. Protein transfection experiments with amyloid fibrils of recombinant HELLF(209-277) or HET-s(218-289) were carried following the general protocol described by Benkemoun et al. (39) with minor modifications. In brief, an agar piece ( $\sim 5 \mathrm{~mm} 3)$ covered with fresh ( $24 \mathrm{~h}$ of growth) prion-free mycelium is placed in a $2-\mathrm{mL}$ screw cap tube containing $500 \mu \mathrm{L}$ of STC buffer ( $0.8 \mathrm{M}$ sorbitol, $50 \mathrm{mM} \mathrm{CaCl} 2,100 \mathrm{mM}$ Tris. $\mathrm{HCl} \mathrm{pH} \mathrm{7.5)} \mathrm{in} \mathrm{addition}$ to $50 \mu \mathrm{L}$ of amyloids ( 2 to $3 \mathrm{mg} \cdot \mathrm{ml}^{-} 1$ ). The mycelium is fragmented using a mechanical cell disruptor (FastPrepTM FP120). Two consecutive runs of $30 \mathrm{~s}$ each at speed of $6 \mathrm{~m} / \mathrm{s}$ were realized and fractions of the suspension (20 to $25 \mu \mathrm{L}$ ) were directly spotted on corn meal medium to be assessed for prion conversion after 4 to $5 \mathrm{~d}$ of regeneration at $26^{\circ} \mathrm{C}$.

Protein Purification. Cells were sonicated on ice in a lysis buffer (Tris $50 \mathrm{mM}$ $150 \mathrm{mM} \mathrm{NaCl}, \mathrm{pH}$ 8) and centrifuged to remove Escherichia coli contaminants Proteins were expressed in inclusion bodies due to their insoluble properties and purified under denaturing conditions. The supernatant was discarded and the pellet incubated with lysis buffer supplemented with $2 \%$ Triton $X-100$. The membrane pellet containing inclusion bodies was extensively washed with lysis buffer to remove Triton X-100 traces and incubated at $60{ }^{\circ} \mathrm{C}$ overnight with $8 \mathrm{M}$ guanidine hydrochloride until complete solubilization. After a centrifugation step at $250,000 \times g$, lysate was recovered and incubated for $2 \mathrm{~h}$ with preequilibrated Ni-NTA beads (Ni Sepharose 6 Fast Flow, GE Healthcare Life Sciences) in a binding buffer (50 mM Tris, $0.5 \mathrm{M}$ $\mathrm{NaCl}, 20 \mathrm{mM}$ imidazole, $7 \mathrm{M}$ urea, $\mathrm{pH}$ 8). Proteins were eluted from the beads with $10 \mathrm{~mL}$ of elution buffer $(50 \mathrm{mM}$ Tris, $0.5 \mathrm{M} \mathrm{NaCl}, 500 \mathrm{mM}$ imidazole, $7 \mathrm{M}$ urea, $\mathrm{pH} 8$ ). After the affinity chromatography step, proteins were loaded on a HiPrep 26/10 desalting column (GE Healthcare) to exchange buffer for $1 \%$ acetic acid and remove low molecular weight compounds. All of the purification steps were realized in $100 \% \mathrm{H}_{2} \mathrm{O}$. It allowed amide proton back exchange under denaturing conditions for the mixed $(1 / 1)\left[\left(U-{ }^{1} H /{ }^{14} \mathrm{~N}\right) /\left(U-{ }^{1} \mathrm{H}_{\mathrm{N}} /{ }^{2} \mathrm{H} /{ }^{15} \mathrm{~N}\right)\right]$ sample.

Assembly of HELLF(209-273) Fibrils In Vitro. The pure protein recovered after high-performance liquid chromatography purification in $1 \%$ acetic acid was concentrated in Amicon Ultra-15 (cutoff $3 \mathrm{kDa}$ ) centrifugal filter units (Merck Millipore) to reach the final protein concentration of $1 \mathrm{mM}$. For the mixed (1/1) $\left[\left(\mathrm{U}-{ }^{1} \mathrm{H} /{ }^{14} \mathrm{~N}\right) /\left(\mathrm{U}-{ }^{1} \mathrm{H}_{\mathrm{N}} /{ }^{2} \mathrm{H} /{ }^{15} \mathrm{~N}\right)\right]$ sample, monomers were solubilized in $1 \%$ acetic acid at $1 \mathrm{mM}$ concentration at a molar ratio of 1:1. Fibrils were formed by adjusting the $\mathrm{pH}$ with $3 \mathrm{M}$ Tris to a $\mathrm{pH}$ value of 7.5. The protein solution was allowed to self-assemble for $2 \mathrm{wk}$ at room temperature under slow shaking. Fibrils were then centrifuged at $20,000 \times g$ and washed several times with water supplemented with $0.02 \% \mathrm{NaN}_{3}$ and transferred to the ssNMR rotor.

Solid-State NMR Spectroscopy of HELLF(209-277). ssNMR spectra were recorded on a $23.5 \mathrm{~T}$ (1 $\mathrm{GHz}{ }^{1} \mathrm{H}$ frequency) spectrometer (Bruker Biospin) equipped with a $0.7-\mathrm{mm}$ triple resonance $\left({ }^{1} \mathrm{H},{ }^{13} \mathrm{C},{ }^{15} \mathrm{~N}\right)$ MAS probe. Sample spinning frequency was $100 \mathrm{kHz}$. Spectra were referenced according to 4,4-dimethyl-4-silapentane-1-sulphonic acid (DSS) signals.

Backbone and Side-Chain Resonances Assignment of HELLF. We used a set of eight 3D ${ }^{1} \mathrm{H}$ detected experiments: $(\mathrm{HCA}) \mathrm{CB}(\mathrm{CA}) \mathrm{NH}[4$ scans, $11 \mathrm{~ms}(\mathrm{t} 3) \times 4 \mathrm{~ms}$ $(\mathrm{t} 2) \times 20 \mathrm{~ms}(\mathrm{t} 1)],(\mathrm{HCO}) \mathrm{CA}(\mathrm{CO}) \mathrm{NH}[16 \mathrm{scans}, 11 \mathrm{~ms}(\mathrm{t} 3) \times 8 \mathrm{~ms}(\mathrm{t} 2) \times 20 \mathrm{~ms}$ (t1)], (H)CANH [4 scans, $11 \mathrm{~ms}(\mathrm{t} 3) \times 8 \mathrm{~ms}(\mathrm{t} 2) \times 20 \mathrm{~ms}(\mathrm{t} 1)$ ], $(\mathrm{H})$ CONH [8 scans, $11 \mathrm{~ms}(\mathrm{t} 3) \times 8 \mathrm{~ms}(\mathrm{t} 2) \times 20 \mathrm{~ms}(\mathrm{t} 1)],(\mathrm{H}) \mathrm{CO}(\mathrm{CA}) \mathrm{NH}[32 \mathrm{scans}, 11 \mathrm{~ms}(\mathrm{t} 3) \times 8 \mathrm{~ms}$ $(\mathrm{t} 2) \times 20 \mathrm{~ms}(\mathrm{t} 1)],(\mathrm{HCA}) \mathrm{CBCAHA}$ [4 scans, $8 \mathrm{~ms}(\mathrm{t} 3) \times 4 \mathrm{~ms}(\mathrm{t} 2) \times 20 \mathrm{~ms}(\mathrm{t} 1)$ ], $(\mathrm{H}) \mathrm{N}(\mathrm{CO}) \mathrm{CAHA}$ [8 scans, $14 \mathrm{~ms}(\mathrm{t} 3) \times 6 \mathrm{~ms}(\mathrm{t} 2) \times 20 \mathrm{~ms}(\mathrm{t} 1)$ ], and $(\mathrm{H}) \mathrm{NCAHA}$ [4 scans, $14 \mathrm{~ms}(\mathrm{t} 3) \times 8 \mathrm{~ms}(\mathrm{t} 2) \times 20 \mathrm{~ms}(\mathrm{t} 1)$ ]. The combination of these experiments allowed the connectivities between each ${ }^{1} \mathrm{H}-{ }^{15} \mathrm{~N}$ couple to intraresidual or sequential $\mathrm{CA}, \mathrm{CA}, \mathrm{CO}$, and $\mathrm{HA}$ resonances, necessary to perform the entire backbone assignment. Side-chain proton assignment was performed using a ${ }^{1} \mathrm{H}_{-}{ }^{13} \mathrm{C}$ cross-polarization (CP)-based sequence followed by a WALTZ mixing step $(\mathrm{H}) \mathrm{CwaltzCH}[2$ scans, $6 \mathrm{~ms}(\mathrm{t} 3) \times 6 \mathrm{~ms}(\mathrm{t} 2) \times 20 \mathrm{~ms}(\mathrm{t} 1)$ ] . We used as a starting point the previously assigned CA and HA chemical shifts to correlate unambiguously all of the carbons and protons of the side chains step by step (27). Spectra were analyzed using the Collaborative Computing Project for NMR (CCPNMR) Analysis Software (40).

Collection of Intra- and Intermolecular Restraints for HELLF from Solid-State NMR. A total of 211 distance restraints per monomer were collected from ${ }^{1} \mathrm{H}$ detected ssNMR spectra to determine the 3D structure of the HELLF(209-273) amyloid fibril. We added to these restraints 34 dihedral angles (phi/psi) estimated from chemical shifts using the TALOS+ software. A total of 143 longrange intramolecular restraints were assigned on 3D $(\mathrm{H}) \mathrm{CHH}$ [4 scans, $6 \mathrm{~ms}$ $(\mathrm{t} 3) \times 4 \mathrm{~ms}(\mathrm{t} 2) \times 20 \mathrm{~ms}(\mathrm{t} 1)], \mathrm{HhNH}[8 \mathrm{scans}, 8 \mathrm{~ms}(\mathrm{t} 3) \times 4 \mathrm{~ms}(\mathrm{t} 2) \times 20 \mathrm{~ms}(\mathrm{t} 1)]$, and $\mathrm{H}(\mathrm{H}) \mathrm{CH}$ [4 scans, $6 \mathrm{~ms}(\mathrm{t} 3) \times 4 \mathrm{~ms}(\mathrm{t} 2) \times 20 \mathrm{~ms}(\mathrm{t} 1)$ ] spectra using a radio frequency-driven recoupling (RFDR) ${ }^{1} \mathrm{H}-{ }^{1} \mathrm{H}$ mixing. A total of 33 intermolecular restraints were assigned on a $3 \mathrm{D} \mathrm{H}(\mathrm{H}) \mathrm{NH}$ spectrum [48 scans, $9 \mathrm{~ms}$ $(\mathrm{t} 3) \times 4 \mathrm{~ms}(\mathrm{t} 2) \times 20 \mathrm{~ms}(\mathrm{t} 1)]$ using a RFDR ${ }^{1} \mathrm{H}-{ }^{1} \mathrm{H}$ mixing, using a mixed $(1 / 1)$ $\left[\left(U-{ }^{1} \mathrm{H} /{ }^{14} \mathrm{~N}\right) /\left(\mathrm{U}-{ }^{1} \mathrm{H}_{\mathrm{N}} /{ }^{2} \mathrm{H} /{ }^{15} \mathrm{~N}\right)\right]$ sample for the unambiguous detection of intermolecular restraints. A RFDR mixing followed by a ${ }^{15} \mathrm{~N}$-edited $\mathrm{CP}$ allowed a magnetization transfer from all of the protons of the $\left(\mathrm{U}-{ }^{1} \mathrm{H} /{ }^{14} \mathrm{~N}\right) \mathrm{HELLF}$ monomers to ${ }^{15} \mathrm{~N}$ atoms of the $\left(\mathrm{U}-{ }^{1} \mathrm{H}_{\mathrm{N}} /{ }^{2} \mathrm{H} /{ }^{15} \mathrm{~N}\right)$, back protonated monomers adjacent in the fibril, followed by the ${ }^{1} \mathrm{H}$ detection of the amide protons.

NMR Structure Calculation of HELLF. The structure of HELLF fibrils was determined in several cycles of structure calculations and restraint analysis with ARIA 2.3 (41). Cross-peak assignments for ${ }^{1} \mathrm{H}-{ }^{1} \mathrm{H}$ correlations were converted into distance restraints with an upper bound of $8.5 \AA$. Backbone dihedral angles were predicted with TALOS+ (30) from ${ }^{1} \mathrm{H},{ }^{13} \mathrm{C}$, and ${ }^{15} \mathrm{~N}$ chemical shifts. TALOS predictions for residues in secondary structure elements $\left(\mathrm{R} 1 \beta_{1-4}, \mathrm{R} 2 \beta_{1-4}\right.$ and $\mathrm{C}$-terminal $\alpha$-helix) were converted in dihedral angle restraints with an error range corresponding to \pm 1.5 times the TALOS error with a minimum of $\pm 15^{\circ}$. HELLF fibril structure was calculated as a pentamer with five copies of HELLF(220-272) using simulated annealing performed with CNS 1.2 (42). The ladder topology was maintained during the calculation through distance restraints ensuring that the distance between equivalent $C_{\alpha}$ atoms in neighboring monomers is constant throughout the pentamer, without fixing a particular distance value, i.e., $d_{m / m+1}=d_{m+1 / m+2}=$ $d_{m+2 / m+3}=d_{m+3 / m+4}=d_{m+4 / m+5}(43,44)$. Additionally, a noncrystallographic symmetry (NCS) restraint was added to minimize the r.m.s.d. between atomic coordinates of the monomers (44). For every ARIA iteration, 100 structures were calculated and the 10 lowest-energy structures from the last iteration were refined in a shell of water molecules (45). On the basis of the identified $\beta$-strands and the in/out distribution of side chains from an initial ARIA calculation using NMR restraints only, intra- and intermonomer hydrogen bond restraints between equivalent $\beta$-strands from the R1 and R2 pseudorepeats were included in subsequent rounds of ARIA calculation. Final restraints and structure statistics are given in SI Appendix, Table S3.

Solid-State NMR Spectroscopy of HEC, HEC, HEC, and HEC ${ }^{\mathbf{\Phi}}$. Proton-detected ssNMR spectra were recorded on a $23.5 \mathrm{~T}\left(1 \mathrm{GHz}{ }^{1} \mathrm{H}\right.$ frequency) and 14.1 spectrometers (Bruker Biospin) equipped with $0.7 \mathrm{~mm}, 1.3 \mathrm{~mm}$, and $3.2 \mathrm{~mm}$ triple resonance $\left({ }^{1} \mathrm{H},{ }^{13} \mathrm{C},{ }^{15} \mathrm{~N}\right)$ MAS probes. Spinning frequency was 
maintained at $100 \mathrm{kHz}(0.7 \mathrm{~mm})$ and $60 \mathrm{kHz}(1.3 \mathrm{~mm})$ for ${ }^{1} \mathrm{H}$ detection and $11 \mathrm{kHz}(3.2 \mathrm{~mm})$ for ${ }^{13} \mathrm{C}$ detection, respectively.

Two-dimensional (2D) (H)NH experiments of HEC and HED were recorded at $100 \mathrm{kHz}$ MAS (1 GHz ${ }^{1} \mathrm{H}$ frequency spectrometer). Resonance assignment of HED, recorded using a $1.3-\mathrm{mm}$ probe $\left(1 \mathrm{GHz}{ }^{1} \mathrm{H}\right.$ frequency spectrometer), was performed using the following set of experiments: $(\mathrm{H}) \mathrm{CONH},(\mathrm{H})(\mathrm{CO}) \mathrm{CA}(\mathrm{CO})$ $\mathrm{NH}$, $(\mathrm{H}) \mathrm{NCAH}$, and $(\mathrm{H}) \mathrm{COCAH}$. Two-dimensional ${ }^{13} \mathrm{C}^{13} \mathrm{C}$ experiments of $\mathrm{HEC} \mathrm{C}^{\mathrm{S}}$ and $\mathrm{HEC}^{\Phi}$ were recorded at $11 \mathrm{kHz}$ MAS $\left(600 \mathrm{MHz}{ }^{1} \mathrm{H}\right.$ frequency spectrometer).

Experimental data were processed using TopSpin and analyzed using Sparky (46) or CcpN CCPNMR.

Modeling of HRAM Family Structures. The 3D models of the PFD of representative proteins of HRAM-2, HRAM-3, and HRAM-4 were constructed with the program MODELER (47) using the HET-s(218-289) (Protein Data Bank 2KJ3) (43) and HELLF(220-272) structures. For each HRAM family, the sequence of the R1 and R2 repeats of HET-s and HELLF were aligned on the predicted repeats of the PFD to be modeled. HRAM models were built as trimers using the atomic coordinates of the R1 and R2 repeats of HELLF and HET-s structures as templates.

1. F. Chiti, C. M. Dobson, Protein misfolding, amyloid formation, and human disease: A summary of progress over the last decade. Annu. Rev. Biochem. 86, 27-68 (2017).

2. S. B. Prusiner, Biology and genetics of prions causing neurodegeneration. Annu. Rev. Genet. 47, 601-623 (2013).

3. D. Eisenberg, M. Jucker, The amyloid state of proteins in human diseases. Cell 148 1188-1203 (2012).

4. J. C. Watts, S. B. Prusiner, $\beta$-Amyloid prions and the pathobiology of Alzheimer's disease. Cold Spring Harb. Perspect. Med. 8, a023507 (2018).

5. S. B. Prusiner, Cell biology. A unifying role for prions in neurodegenerative diseases. Science 336, 1511-1513 (2012).

6. D. W. Sanders et al., Distinct tau prion strains propagate in cells and mice and define different tauopathies. Neuron 82, 1271-1288 (2014).

7. N. L. Rey et al., $\alpha$-Synuclein conformational strains spread, seed and target neurona cells differentially after injection into the olfactory bulb. Acta Neuropathol. Commun. 7, 221 (2019).

8. J. Collinge, A. R. Clarke, A general model of prion strains and their pathogenicity Science 318, 930-936 (2007).

9. W. Qiang, W.-M. Yau, J.-X. Lu, J. Collinge, R. Tycko, Structural variation in amyloid- $\beta$ fibrils from Alzheimer's disease clinical subtypes. Nature 541, 217-221 (2017).

10. R. Morales, I. Moreno-Gonzalez, C. Soto, Cross-seeding of misfolded proteins: Implications for etiology and pathogenesis of protein misfolding diseases. PLoS Pathog. 9 e1003537 (2013).

11. B. Ren et al., Fundamentals of cross-seeding of amyloid proteins: An introduction J. Mater. Chem. B Mater. Biol. Med. 7, 7267-7282 (2019).

12. T. R. Sampson et al., A gut bacterial amyloid promotes $\alpha$-synuclein aggregation and motor impairment in mice. elife 9, e53111 (2020).

13. I. Horvath, P. Wittung-Stafshede, Cross-talk between amyloidogenic proteins in type2 diabetes and Parkinson's disease. Proc. Natl. Acad. Sci. U.S.A. 113, 12473-12477 (2016).

14. K. H. Lim, Diverse misfolded conformational strains and cross-seeding of misfolded proteins implicated in neurodegenerative diseases. Front. Mol. Neurosci. 12, 158 (2019).

15. C. Ritter et al., Correlation of structural elements and infectivity of the HET-s prion Nature 435, 844-848 (2005).

16. C. Wasmer et al., Amyloid fibrils of the HET-s(218-289) prion form a beta solenoid with a triangular hydrophobic core. Science 319, 1523-1526 (2008)

17. E. Vázquez-Fernández et al., The structural architecture of an infectious mammalian prion using electron cryomicroscopy. PLoS Pathog. 12, e1005835 (2016).

18. A. W. P. Fitzpatrick et al., Cryo-EM structures of tau filaments from Alzheimer's disease. Nature 547, 185-190 (2017)

19. A. Daskalov et al., Signal transduction by a fungal NOD-like receptor based on propagation of a prion amyloid fold. PLoS Biol. 13, e1002059 (2015).

20. R. Riek, S. J. Saupe, The HET-S/s prion motif in the control of programmed cell death. Cold Spring Harb. Perspect. Biol. 8, a023515 (2016).

21. S. J. Saupe, The [Het-s] prion of Podospora anserina and its role in heterokaryon incompatibility. Semin. Cell Dev. Biol. 22, 460-468 (2011).

22. C. Seuring et al., The mechanism of toxicity in HET-S/HET-s prion incompatibility. PLoS Biol. 10, e1001451 (2012).

23. J. Greenwald et al., The mechanism of prion inhibition by HET-S. Mol. Cell 38, 889-899 (2010)
Data Availability. All data from this work are included in the main text and SI Appendix.

ACKNOWLEDGMENTS. We acknowledge financial support from the European Research Council (ERC) under the European Unions Horizon 2020 Research and Innovation Programme (ERC-2015-CoG GA 648974 to G.P. and ERC-2015-StG GA 639020 to A.L.), IdEx Bordeaux (Chaire d'Installation to B.H., ANR-10-IDEX-03-02), the Agence Nationale de la Recherche (ANR) (ANR-14-CE09-0020-01 to A.L., ANR-13-PDOC-0017-01 to B.H. and ANR-17CE11-0035 to S.J.S), the INCEPTION project (Programme d'investissements d'avenir/ANR-16-CONV-0005) and the CNRS (IR-RMN FR3050). J.S. and L.B.A. were supported by individual Marie Sklodowska-Curie incoming fellowships (grant agreements 661799 "COMPLEX-FAST-MAS" and 624918 "MEM-MAS"). A.D. was supported by the Nouvelle Aquitaine Regional Council. We thank the Nouvelle Aquitaine Regional Council, University of Bordeaux, and the Contrat Plan Etat-Region (CPER) CampusB Bordeaux for the acquisition of the NMR equipment. This work has benefited from the Biophysical and Structural Chemistry Platform at IECB, CNRS UMS 3033 INSERM US001.

24. A. Loquet, S. J. Saupe, Diversity of amyloid motifs in NLR signaling in fungi. Biomolecules 7, 38 (2017)

25. A. Daskalov, W. Dyrka, S. J. Saupe, Theme and variations: Evolutionary diversification of the HET-s functional amyloid motif. Sci. Rep. 5, 12494 (2015).

26. V. Agarwal et al., De novo 3D structure determination from sub-milligram protein samples by solid-state $100 \mathrm{kHz}$ MAS NMR spectroscopy. Angew. Chem. Int. Ed. Engl. 53, 12253-12256 (2014)

27. L. B. Andreas et al., Structure of fully protonated proteins by proton-detected magic angle spinning NMR. Proc. Natl. Acad. Sci. U.S.A. 113, 9187-9192 (2016).

28. A. Samoson, H-MAS. J. Magn. Reson. 306, 167-172 (2019).

29. J. Stanek et al., NMR spectroscopic assignment of backbone and side-chain protons in fully protonated proteins: Microcrystals, sedimented assemblies, and amyloid fibrils. Angew. Chem. Int. Ed. Engl. 55, 15504-15509 (2016).

30. Y. Shen, F. Delaglio, G. Cornilescu, A. Bax, TALOS+: A hybrid method for predicting protein backbone torsion angles from NMR chemical shifts. J. Biomol. NMR 44, 213-223 (2009)

31. A. Loquet, K. Giller, S. Becker, A. Lange, Supramolecular interactions probed by 13C13 C solid-state NMR spectroscopy. J. Am. Chem. Soc. 132, 15164-15166 (2010).

32. B. Rost, Twilight zone of protein sequence alignments. Protein Eng. 12, 85-94 (1999)

33. M. Olivella, A. Gonzalez, L. Pardo, X. Deupi, Relation between sequence and structure in membrane proteins. Bioinformatics 29, 1589-1592 (2013).

34. A. Daskalov et al., Contribution of specific residues of the $\beta$-solenoid fold to HET-s prion function, amyloid structure and stability. PLoS Pathog. 10, e1004158 (2014).

35. W. Wan, G. Stubbs, Fungal prion HET-s as a model for structural complexity and selfpropagation in prions. Proc. Natl. Acad. Sci. U.S.A. 111, 5201-5206 (2014).

36. A. V. Kajava, A. C. Steven, Beta-rolls, beta-helices, and other beta-solenoid proteins. Adv. Protein Chem. 73, 55-96 (2006).

37. B. Falcon et al., Structures of filaments from Pick's disease reveal a novel tau protein fold. Nature 561, 137-140 (2018)

38. B. Falcon et al., Novel tau filament fold in chronic traumatic encephalopathy encloses hydrophobic molecules. Nature 568, 420-423 (2019).

39. L. Benkemoun et al., Methods for the in vivo and in vitro analysis of [Het-s] prion infectivity. Methods 39, 61-67 (2006).

40. T. J. Stevens et al., A software framework for analysing solid-state MAS NMR data. J. Biomol. NMR 51, 437-447 (2011).

41. B. Bardiaux, T. Malliavin, M. Nilges, ARIA for solution and solid-state NMR. Methods Mol. Biol. 831, 453-483 (2012).

42. A. T. Brunger, Version 1.2 of the crystallography and NMR system. Nat. Protoc. 2 2728-2733 (2007)

43. H. Van Melckebeke et al., Atomic-resolution three-dimensional structure of HETs(218-289) amyloid fibrils by solid-state NMR spectroscopy. J. Am. Chem. Soc. 132 13765-13775 (2010)

44. M. Nilges, A calculation strategy for the structure determination of symmetric dimers by 1H NMR. Proteins 17, 297-309 (1993).

45. J. P. Linge, M. A. Williams, C. A. E. M. Spronk, A. M. J. J. Bonvin, M. Nilges, Refinement of protein structures in explicit solvent. Proteins 50, 496-506 (2003)

46. W. Lee, M. Tonelli, J. L. Markley, NMRFAM-SPARKY: Enhanced software for biomolecular NMR spectroscopy. Bioinformatics 31, 1325-1327 (2015)

47. A. Sali, T. L. Blundell, Comparative protein modelling by satisfaction of spatial re straints. J. Mol. Biol. 234, 779-815 (1993). 